\section{Luis David Castiel' \\ Carlos Álvarez-Dardet"}

\title{
La salud persecutoria
}

\section{Persecutory health}

\section{RESUMEN}

Se plantea una aproximación crítica desde el punto de vista de la filosofía social para analizar aspectos de los sistemas vigentes de producción de conocimientos y tecnologías en promoción de la salud. Se analisan sus capacidades para generar perplejidades, dilemas e insatisfacciones, aunque se admita su potencia y efectividad en muchos niveles. Para ello se busca apoyo en la noción de "iatrogenia social" de Ivan Illich, y en la idea del "salutismo coercitivo", de Peter Skrabanek. También se apela al trabajo de Giorgio Agamben y su teorización del "homo sacer", cuya existencia no merecía ser vivida. La discusión enfoca los límites y los dilemas del empleo de la idea de responsabilidad individual en términos de su aplicación como técnica de persuasión en los discursos y prácticas de la promoción de la salud, que con sus propuestas generan la "salud persecutoria" como efecto secundario.

DESCRIPTORES: Filosofía. Promoción de la salud. Responsabilidad Social. Responsabilidad legal. Responsabilidad civil.

\begin{abstract}
A critical approach from the standpoint of social philosophy is taken aiming at analyzing aspects of current production of knowledge and technologies in health promotion. Its capacity of generating perplexities, dilemmas and dissatisfactions is also evaluated, despite its strength and effectiveness in many levels. The analysis is based on Ivan Illich's "social iatrogenesis," Peter Skrabanek's "coercive healthism," and Giorgio Agamben's theoretical approach of "homo sacer" - a man whose life is worthless. The limits and dilemmas of the idea of personal responsibility are discussed as a persuasion strategy in health promotion discourses and practices. All these proposals produce "persecutory health" as a side effect.
\end{abstract}

Quantitativos em Saúde. Escola Nacional de Saúde Pública. Fundação Oswaldo Cruz. Rio de Janeiro, Brasil

" Observatorio de Políticas Públicas y Salud. Departamento Enfermería Comunitaria. Universidad de Alicante. Alicante, España

\section{Correspondência | Correspondence:} Luis David Castiel

Departamento de Epidemiologia

Escola Nacional de Saúde Pública

Fundação Oswaldo Cruz

Rua Leopoldo Bulhões 1480 sala 802

21041-210 Rio de Janeiro, RJ, Brasil

E-mail: luis.castiel@ensp.fiocruz.br

KEYWORDS: Philosophy. Health promotion. Social responsibility. Liability, legal. Damage liability. 
Hay que se enfatizar la importancia de una responsabilidad individual que respete la autonomía de las personas, tanto en la contabilidad de derechos como de deberes en mezcla con la responsabilidad colectiva, según los problemas a considerar en la gestión de la salud. ${ }^{21,22} \mathrm{El}$ punto de vista de este trabajo se sitúa en la perspectiva de prevención cuaternaria ${ }^{12}$ en términos de desarrollar acciones de moderación aplicadas al énfasis de control de comportamientos en contra de los innumerables riesgos a la salud.

Hay críticas a las exageradas reivindicaciones en relación a la dudosa efectividad preventiva de la promoción de salud, denominada por Le Fanu como preventivitis. ${ }^{16}$ La prevención se ha vuelto un elemento obsesivo en el campo de la salud y parece más bien ser una de las manifestaciones que acompaña el ambiente de alta desconfianza, incertidumbre e inseguridad que afecta el mundo moderno.

En este sentido, debemos hablar de las actuales precariedades de los excesos ${ }^{6}$ de la prevención en salud. Este es un terreno en el cual tendremos que considerar quizás algo como una metaprevención - o sea la prevención de la hiperprevención generada por la dimensión cada vez más persecutoria de las estrategias vigentes de promoción y prevención en las actuales prácticas de salud.

Existe algo de persecutorio en la idea de conocer el futuro para prevenirse de las muchas amenazas que parecen acecharnos. Esta preocupación aparece cada vez más en las ciencias que tienen el apremio en relación con la predictibilidad que caracteriza otras disciplinas científicas, como la economía o la epidemiología (incluso, ambas operan con la noción virtual del riesgo). Además, hay sectores de las ciencias de la salud que empezaron a colaborar con más fuerza con estrategias corporativas de la industria farmacéutica para hacer la ocurrencia de la enfermedad como un espacio ampliable tanto en el presente al crear nuevas enfermedades ${ }^{19}$ y mediante la noción del riesgo, medicalizando también el futuro. ${ }^{13}$ La noción del riesgo acompaña al encogimiento de sentimiento de la capacidad de gestionar los muchos elementos y variables que componen el tiempo presente $^{10}$ y se manifiesta en las sensaciones de desasosiego que rondan a las sociedades modernas. Esta sensación de gran inseguridad de nuestra época parece generar una búsqueda de previsibilidad en relación a los escenarios y hechos que puedan ocurrirnos. Hay muchos dispositivos predictivos desarrollados por las tecnociencias, bajo sus cánones de racionalidad. Paradójicamente, somos testigos de que la racionalidad no trae obligatoriamente certidumbre, confianza, tranquilidad..$^{15}$

Quizás, la gran disponibilidad de herramientas de desarrollo de modelos matemáticos y el gran énfasis en técnicas estadísticas prospectivas sean manifestaciones emblemáticas de este estado de cosas, como síntoma de la necesidad de control del futuro. Sin dudas el afán predictivo se ha acentuado bastante, de tal manera que algunas ciencias hightech presentan una aura futurista que las aproxima mucho a las narrativas de la ciencia-ficción. Por ejemplo, la sensación de paranoia y ansiedad generada por el riesgo ${ }^{9}$ de una pandemia de gripe humana antes incluso del cambio del virus aviar para generar el contagio persona-persona. Además, no deja de ser una trágica ironía el hecho de ser aves migratorias las responsables de la difusión del virus H5N1 sin respetar fronteras geopolíticas cuando los países de economía avanzada intentan frenar la entrada incontrolada de inmigrantes en sus tierras.

Por otra parte, los discursos acerca de la salud significan modos de pensar, escribir, hablar acerca de la salud y sus prácticas. Es necesario ubicar los discursos en determinados momentos históricos y conocer las razones por las que se legitiman al acompañar y ajustarse al orden económico, político y social donde son generados, sostenidos y reproducidos. ${ }^{24}$ Los discursos acerca de la salud y los riesgos son construcciones contingentes, de carácter normativo, inapelablemente vinculadas a intereses corporativos y económicos. Además, dependen, de manera explícita o no, de definiciones de lo que sea el ser humano, el tipo de sociedad que se busca y de las maneras conseguirlo. ${ }^{4}$

Actualmente, predominan discursos acerca de la salud producidos por la salud pública hegemónica. Se utilizan conceptos y estrategias como promoción y educación para la salud, marketing social, screening (tamizaje) diagnóstico, vacunación, participación de la comunidad, políticas públicas de salud, colaboración intersectorial, ecología, economía de la salud. En todos estos sectores la obtención de datos epidemiológicos es vital para producir las evidencias en las cuales se deben basar las decisiones en salud. ${ }^{17}$

La idea de que la verdad solamente puede ser obtenida bajo búsquedas casi paroxísticas de las evidencias - un hábil término de este discurso de la verdad, de lo que es lo empiricamente correcto o sea, de lo que parece ser tangible, pues lo que no es retenido bajo este filtro, o tiene importancia secundaria, o peor, no debería existir. Una de las críticas al enfoque de las evidencias es que las premisas filosóficas vinculadas al empiricismo evidenciológico asumen la insostenible proposición de que las observaciones puedan ser hechas de modo totalmente objetivo, independientemente de teorías y de la visión de mundo del observador. ${ }^{2}$

Es importante señalar la noción de responsabilidad que predomina en muchos discursos de salud. Esta cuestión admite acercamientos éticos, filosóficos y jurídicos que, a pesar de su importancia, no serán discutidos en este momento. La idea de responsabilidad, en términos generales, vinculase a la noción de obligación de individuos $\mathrm{y} / \mathrm{o}$ instituciones de responder a instancias de vigilancia y control por determinadas acciones, y re- 
lativas a objetos que, por medio de algún compromiso, están bajo la confianza de alguien. ${ }^{18}$ La responsabilidad consiste en una idea normativa que permite pactos esenciales para la organización de los colectivos humanos. Es inevitable la asociación de "responsabilidad" con "culpa", especialmente cuando las obligaciones no se cumplen. La retórica de la responsabilidad personal y de los cambios de conducta que predominan en la promoción de la salud ocurre es coetánea del ambiente individualista y moralista de las sociedades post-modernas y de las crisis fiscales y económicas de los sistemas de bienestar social de muchas naciones.

A pesar de su buen desarrollo en la literatura de promoción de la salud, ${ }^{5,11}$ no hay cambios significativos en la énfasis acerca de la responsabilidad de las personas en las prácticas individuales de promoción de la salud. De todas maneras, la discusión sobre responsabilidad individual recibió una elaboración importante por parte de Ivan Illich.* Este autor es conocido en el mundo de la salud por su crítica a los sistemas de salud en los años 70, la Némesis Médica ${ }^{14}$ - una referencia que no cesa de volver para diagnosticar las cuestiones del mercadeo de la enfermedad o sea las acciones del complejo médicoindustrial que resultan en la ampliación de los límites de lo que sean las condiciones de salud tratables.

En el texto "Salud como responsabilidad de cada uno - no, gracias!" basado en una conferencia pronunciada en Hannover (Alemania), Illich* explica como su "no gracias!" se debe a cinco razones:

1- Por no considerar probada la extendida idea de una incapacidad de las personas legas para tomar decisiones sobre su propia salud, el paternalismo profesional;

2- Por no considerar que exista una supuesta escasez de recursos para la curación, sino por entender que muchos individuos hoy día mueren de hambre, ideología de la escasez;

3- Por asumir que no hay controles satisfactorios sobre contaminantes $\mathrm{u}$ otros agentes $\mathrm{y}$ objetos nocivos para la salud de humanos y animales, pensamiento sistémico;

4- Por criticar la ética de la responsabilidad en salud porque tanto las enfermedades como la presencia de muerte pueden enseñar formas de autoconocimiento, $y$ porque entiende una falta de respeto la aceptación de la inevitables epidemias de la era post industrial como una forma superior de salud, psicología liberadora;

5- porque han sido destruidos los espacios culturales, técnicos y arquitectónicos de muchas poblaciones que, de esta manera, se alienan de sus artes heredadas relacionadas con el sufrimiento y la muerte, el cliché de que no existe esperanza de vuelta atrás para las consecuencias de los mecanismos modernos de desarrollo.

Esta destrucción de los recursos y los activos sociales para la salud es lo que Illich consideraba iatrogenia social. ${ }^{14}$ Una de las consecuencias de la medicalización de los futuros que estamos viviendo es la aparición de expansiones temporales de la noción de iatrogenia. Ya no solo pueden aparecer efectos secundarios del tratamiento de enfermedades reales sino que aparece la iatrogenia virtual. Los médicos no solo diagnostican y tratan enfermedades, cada vez mas diagnostican y tratan riesgos. Como quiera que los riesgos no siempre ocurren, nos encontramos en una nueva situación en la práctica medica, que incluye los efectos secundarios adversos del diagnostico y del tratamiento preventivo de enfermedades que nunca ocurrirían.

El contrato implícito en la relación médico-paciente se establecía clásicamente después de una petición de ayuda demandada explícitamente por parte del enfermo (autonomía), bajo la aceptación de los principios de beneficencia y no-maleficencia. Sin embargo, una gran parte de la demanda sanitaria en la actualidad es inducida por la medicalización de los futuros y la medicina del riesgo, circunstancia que claramente afecta a la autonomía de los pacientes. El hecho de que los médicos diagnostiquen y traten riesgos virtualiza la noción de beneficencia. Si alguien después de haber sido tratado para reducir los niveles de colesterol e hipertensión no desarrolla infarto, ¿Puede este hecho ser atribuido con certeza a la acción médica en este caso concreto? Los médicos en la actualidad obtienen crédito de prevenir dolencias que nunca ocurrirían. Lo mismo sucede con los límites del principio de no-maleficencia ¿Son admisibles los efectos secundarios del diagnostico y el tratamiento de las enfermedades que no ocurrirán? ¿El nivel de daño físico, mental o económico debe ser el mismo para diagnosticar y tratar riesgos que para diagnosticar y tratar enfermedades?

Además, para Illich ${ }^{14}$ el concepto de salud de la modernidad europea representó una quiebra con la tradición Galénica para la que la salud consistía en una fusión armónica, en un orden estable y en una interacción equilibrada de los elementos básicos. A partir del siglo XVII, los intentos de controlar la naturaleza resituarán el ideal de salud como busca de armonía con el cosmos en una línea de progresiva apariencia de manipulación.

Desde esta premisa, la salud como "algo que se poseía" adquirió aceptación desde el ultimo cuarto del siglo XVIII. En el XIX y en los inicios del siglo pasado, se volvió normal designar el cuerpo y la salud con pronombres posesivos, como "tu" cuerpo o "mi" salud. Si en esta época tenia sentido hablar de salud y

* Illich I. Health as one's own responsibility -No, thank you! Londres;1993. Disponible en: http://www.pudel.uni-bremen.de/pdf/Illich_1429id. pdf [Acceso en 28 mar 2007] 
responsabilidad como ideales dignos de crédito, ahora estas nociones normativas parecen desfasadas y actúan mas bien como elementos desorientadores, o tan solo como argumentos moralmente coercitivos.

A pesar del característico tono provocador y categórico del discurso illichiano, es importante tener acceso a pensadores que han procurado desestabilizar conceptos consagrados para buscar la mejor comprensión posible de las complejas configuraciones, presupuestos e insuficiencias de muchos modelos y teorías prevalentes en la sanidad occidental.

Otro abordaje agudo y muy pertinente en este sentido fue desarrollado por Petr Skrabanek, ${ }^{25}$ medico checo radicado en Dublín después de la Primavera de Praga en 1968 y que fue uno de los fundadores de la Red Internacional de Escépticos con el Colesterol. Skrabanek hizo una crítica magistral a la propuestas dominantes en la promoción de la salud individualista a partir del "salutismo", no como necesidad de la personas sino como un componente de una doctrina de Estado y de "estilismo de vida". Es una ideología sustentada por la biomedicina en la que se atribuye una gran parte de la responsabilidad en la enfermedad a los comportamientos no saludables. ${ }^{19,20}$

De la unión de estas dos perspectivas surge la "salud coercitiva" que pervertiría los ideales de la "medicina con rostro humano". Los promotores de la salud actuales serian los sacerdotes de la salud anticipatoria, que apelan mediante la referencia obsesiva a los factores de riesgo - algo que conlleva a constantes escudriñamientos de las condiciones de salud por medio de tests para detectarlos y de prescripciones comportamentales para controlarlos. ${ }^{25}$

Los temas de la medicalización, del salutismo y de la culpabilización en la salud fueron también desarrollados por Crawford hace alrededor de trés décadas. ${ }^{7,8}$ Pero, el tema vuelve a preocuparnos porque hay que considerar el énfasis moralista actual en las complejas sociedades modernas y su preocupación por la responsabilidad y la atribución de culpa. La mirada moralista se orienta por razonamientos que buscan el establecimiento de causas bien definidas, procura ubicar y castigar a los responsables/culpables por sus fallos.

No es frecuente en estas circunstancias que exista mucha disponibilidad para los beneficios de la duda en relación a la indefinición de las causas efectivas o de la culpabilidad de los acusados. En verdad, en tiempos en los cuales la idea probabilística del riesgo es dominante, la culpa está más presente y sobreviene antes de los hechos indeseables - basta con estar supuestamente expuesto a los innumerables riesgos enunciados por los discursos de la prevención. Un estudio cualitativo hecho en Escocia ha enseñado que la posibilidad de atribución de culpa por parte de los médicos puede impedir que las personas busquen atención médica en situaciones potencialmente graves de dolor torácico. ${ }^{23}$

El principio de la prevención/precaución se está volviendo cada vez más imperativo en tiempos en los cuales la consideración de escenarios futuros es una constante en las propuestas de gestión de varios aspectos de la vida. La prevención de los riesgos tiene sus ambivalencias, según juicios eventualmente imponderables - pueden suponer medidas expectantes o acciones urgentes. El principio de la prevención/precaución puede ser utilizado para manipular situaciones, de acuerdo con las circunstancias y los intereses en cuestión. Solamente después que el futuro se vuelve presente, es posible saber si las especulaciones de antelación se han confirmado o si hubo un error de evaluación de probabilidades. ${ }^{15}$

En el caso de la prevención de los riesgos para la salud, frente a tantas contingencias relativas a la determinación causal y la manifestación de enfermedades, aunque se tomen todas las medidas preventivas posibles, no tenemos certidumbre de que los resultados de protección nos sean garantizados en función de tales medidas. Debemos enfatizar que no se está depreciando tontamente el poder del conocimiento disponible producido acerca del riesgo en salud, eso es indudablemente importante en relación al conjunto de prácticas efectivas para la prevención de enfermedades. Lo que queremos señalar son los posibles efectos de exageraciones en su utilización indiscriminada y sus vínculos con aspectos socioculturales indeseables relativos al individualismo y el control de las personas. Este es un efecto secundario de la promoción de la salud, llamado "salud persecutoria". Como si el camino responsable para una buena salud dependiera esencialmente de acciones responsables individuales, sin cambios en el importante nivel de responsabilidad de empresas, de instituciones y en las relaciones políticas y económicas entre países. ${ }^{26}$

Los cálculos de los riesgos, en términos de una orientación futurológica, tienen un importante papel en el sentido de establecer regularidades y patrones hasta el punto que sea posible la producción de previsibilidad para el control y la prevención. Los discursos acerca del riesgo ilustran la ambivalencia en varios niveles: de una parte tenemos un alto nivel de inseguridad emocional colectiva en relación a los innumerables riesgos actuales como enseñan las investigaciones científicas; al mismo tiempo, existe una aparente primacía de la idea de certidumbre propuesta por los dispositivos racionales de la ciencia moderna. ${ }^{3}$

La idea de sobrevida, sin cualquier dimensión relativa a la capacidad de acción política en búsqueda de cambios estructurales es una de las tesis más conocidas de Giorgio Agamben. ${ }^{1}$ Este tema acompaña su teorización acerca del homo sacer - la impresionante figura del derecho romano antiguo que denominaba de esta 
manera al individuo cuya existencia no era digna de ser vivida, ni siquiera como ofrenda o como sacrificio ritual a los dioses. Pero que podría ser eliminado, sin que este evento fuera encarado como crimen, pues su derecho a la vida no estaba previsto por la ley. Vale la pena mencionar que la palabra latina "sacer" posee una curiosa ambigüedad semántica, quizás signo de la ambivalencia ya mencionada: puede significar tanto "sagrado", "santificado", como "maldito", "dedicado a la destrucción", "horrible".**

Quizás no sea absurdo indicar que, para aquellos que pueden actuar como agentes de consumo, una de las formas de pensar las prácticas de salud como apoyando la producción de una mercancía que podemos llamar sobrevida desnuda. Esto tiene la finalidad de generar individuos cuya finalidad más importante en su vida sea su propia supervivencia. Podemos considerar que la promoción de la salud actual enfatiza ciertas prácticas individualistas y apolíticas de búsqueda de la salud. El bien supremo de la vida es prolongar la vida en términos de longevidad, de preferencia con el máximo de confort que sea posible obtener conforme las circunstancias de la vida. La promoción de la salud hegemónica puede ser interpretada como una política persecutoria de neohigiene, de fuerte contenido moral con vistas a la longevidad, bajo la perspectiva de que nos mantengamos en el interior de la economía globalizada de acuerdo con la perspectiva del mundo racional de la productividad, de la generación de riquezas y del consumo. Además, procurando el imposible equilibrio esquizofrénico entre la búsqueda de satisfacción de deseos generados por las múltiples tentaciones existentes al nuestro alrededor y una moderación responsable para que no seamos consumidos en este proceso.

** Ultralingua. Ultralingua and Collins Dictionaries for French English, Spanish English, Italian English; Gram [dicionário online]. Mineápolis: Ultralingua, Inc.; 1997. Disponible en: http://www.ultralingua.com/onlinedictionary/ [Acceso en 28 mar 2006] 


\section{REFERENCIAS}

1. Agamben G. Homo Sacer. Poder soberano e vida nua Belo Horizonte: Ed. da UFMG, 2002.

2. Barata RB. Epidemiologia e saber científico. Rev Bras Epidemiol. 1998;1(1):14-27.

3. Bauman Z. Modernity and Ambivalence. Oxford: Polity Press; 1991.

4. Buchanan DR. An ethic for health promotion. Rethinking the sources of human well-being. Oxford: Oxford University Press; 2000.

5. Buck C. Beyond Lalonde: Creating health. Can J Public Health. 1985;76(Supl 1):19-24.

6. Castiel LD, Alvarez-Dardet C. Las tecnologías de la información y la comunicación en salud pública: las precariedades del exceso. Rev Esp Salud Publica. 2005;79(3):331-7.

7. Crawford R. You are dangerous to your health: the ideology and politics of victim blaming. Int I Health Serv. 1977;7(4):663-80.

8. Crawford R Healthism and the medicalization of everyday life. Int J Health Serv.1980;10(3):365-88.

9. Crawford R. Risk ritual and the management of control anxiety in medical culture. Health (London). 2004;8(4):505-28.

10. Cruz M. Las malas pasadas del pasado. Identidad, responsabilidad, historia. Barcelona: Anagrama; 2005.

11. Downie RS, Tannahill C, Tannahill A. Health promotion Models and values. 2.ed. Oxford: Oxford University Press; 1996.

12. Gérvas J. Moderación en la actividad médica preventiva y curativa. Cuatro ejemplos de necesidad de prevención cuaternaria en España. Gac Sanit. 2006;20(Supl I):127-34.

13. Gøtzsche PC. Commentary: Medicalisation of risk factors. BMJ.2002;324(7342):890-1.

14. Illich I. Medical Nemesis: The expropriation of health, London: Marian Boyars; 1975.
15. Innerarity D. La sociedad invisible. Madrid: Ed. Espasa Calpe; 2004.

16. Le Fanu J. Does health education work? In: Le Fanu J, editor. Preventionitis: The exaggerated claims of health promotion. Londres: The Social Affairs Unit;1994. p. 89-105.

17. Lupton D. The imperative of health. Public health and the regulated body. London: Sage;1995.

18. Martin MW. Responsibility for health and blaming victims. J Med Humanit. 2001; 22(2):95-114.

19. Moynihan R, Heath I, Henry D. Selling sickness: the pharmaceutical industry and disease mongering. BMJ. 2002;324(7342):886-91.

20. Moynihan R, Cassels A. Selling sickness: How the worlds biggest pharmaceutical companies are turning us all into patients. New York: Nation Books; 2005.

21. Palomo L, Ortún V, Benavides FG, Marquez-Calderón S. La salud pública frente a los efectos secundarios del progreso. Gac Sanit. 2006; 20(Supl 1):209-21.

22. Reith G. Uncertain times. The notion of 'risk' and the development of modernity. Time Soc. 2004;13(2):383402.

23. Richards H, Reid M, Watt G. Victim-blaming revisited: a qualitative study of beliefs about illness causation, and responses to chest pain. Fam Pract. 2003;20(6):711-6.

24. Robertson A. Biotechnology, political rationality and discourses on health risk. Health.2001;5(3)293-309.

25. Skrabanek P. The death of humane medicine and the rise of coercive healthism. London: Social Affairs Unit; 1994.

26. Van de Vathorst S, Alvarez-Dardet C. Doctors as judges: the verdict on responsibility for health. J Epidemiol Community Health. 54(3):162-4, 2000. 